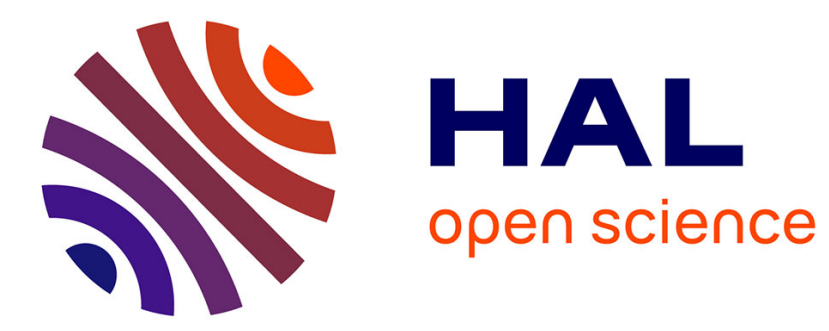

\title{
Effects of steady wake-jets on subcritical cylinder flow
} Donglai Gao, Guanbin Chen, Wenli Chen, Yewei Huang, Hui Li

\section{To cite this version:}

Donglai Gao, Guanbin Chen, Wenli Chen, Yewei Huang, Hui Li. Effects of steady wake-jets on subcritical cylinder flow. Experimental Thermal and Fluid Science, 2019, 102, pp.575-588.

10.1016/j.expthermflusci.2018.12.026 . hal-02558435

\section{HAL Id: hal-02558435 \\ https://hal.science/hal-02558435}

Submitted on 28 May 2020

HAL is a multi-disciplinary open access archive for the deposit and dissemination of scientific research documents, whether they are published or not. The documents may come from teaching and research institutions in France or abroad, or from public or private research centers.
L'archive ouverte pluridisciplinaire HAL, est destinée au dépôt et à la diffusion de documents scientifiques de niveau recherche, publiés ou non, émanant des établissements d'enseignement et de recherche français ou étrangers, des laboratoires publics ou privés. 


\section{Accepted Manuscript}

Effects of steady wake-jets on subcritical cylinder flow

Donglai Gao, Guanbin Chen, Wenli Chen, Yewei Huang, Hui Li

PII: $\quad$ S0894-1777(18)31300-1

DOI: $\quad$ https://doi.org/10.1016/j.expthermflusci.2018.12.026

Reference: $\quad$ ETF 9691

To appear in: $\quad$ Experimental Thermal and Fluid Science

Received Date: $\quad 27$ July 2018

Accepted Date: 21 December 2018

Please cite this article as: D. Gao, G. Chen, W. Chen, Y. Huang, H. Li, Effects of steady wake-jets on subcritical cylinder flow, Experimental Thermal and Fluid Science (2018), doi: https://doi.org/10.1016/j.expthermflusci. 2018.12.026

This is a PDF file of an unedited manuscript that has been accepted for publication. As a service to our customers we are providing this early version of the manuscript. The manuscript will undergo copyediting, typesetting, and review of the resulting proof before it is published in its final form. Please note that during the production process errors may be discovered which could affect the content, and all legal disclaimers that apply to the journal pertain. 


\title{
Effects of steady wake-jets on subcritical cylinder flow
}

\author{
Donglai Gao ${ }^{\mathrm{a}, \mathrm{b}, \mathrm{c}}$, Guanbin Chen ${ }^{\mathrm{a}, \mathrm{b}}$, Wenli Chen ${ }^{\mathrm{a}, \mathrm{b}, *}$, Yewei Huang ${ }^{\mathrm{a}, \mathrm{b}}, \mathrm{Hui}_{\mathrm{Li}}^{\mathrm{a}, \mathrm{b}}$ \\ ${ }^{a}$ Key Lab of Smart Prevention and Mitigation of Civil Engineering Disasters of the Ministry of Industry and Information \\ Technology, Harbin Institute of Technology, Harbin, 150090 China \\ ${ }^{b}$ Key Lab of Structures Dynamic Behavior and Control of the Ministry of Education, Harbin Institute of Technology, Harbin, \\ 150090 China \\ ${ }^{c}$ Aix-Marseille Univ, CNRS, Centrale Marseille, IRPHE, Marseille, 13013 France
}

\begin{abstract}
We investigate the effects of active wake-jets (characterized by a dimensionless jet momentum coefficient $C_{\mu}$ ) on the suppression of aerodynamic forces and the manipulation of wake flow topology behind a cylindrical model through wind tunnel tests. The active jets are positioned at the rear stagnation points of the cylindrical test model. The experimental campaign is conducted at a subcritical Reynolds number of $\operatorname{Re}=3.33 \times 10^{4}$. The surface pressure distributions around the bare and controlled cylinders are obtained by using a pressure measurement system. Apart from pressure measurements, we also obtain the streamwise and spanwise flow structures around the circular cylinder with different $C_{\mu}$ (including $C_{\mu}=0$ ) by employing the particle image velocimetry (PIV) technique. Pressure measurement results demonstrate that the lift force acting on the cylindrical test model is greatly reduced and drag decreased with the implementation of active wake-jets. Besides, it is found that a higher $C_{\mu}$ contributes to a better control effectiveness in unsteady lift forces but not necessarily a better drag reduction. PIV measurement results indicate that the mechanism of the active jet control scheme is to impose steady and symmetric perturbations into the unsteady and asymmetric flows in the cylinder wake. Owing to the dynamic competition of the wake-jet flow and shear layer flows, the vortex shedding pattern behind the controlled cylinder is greatly modified, vortex formation length significantly elongated and the fluctuations of aerodynamic forces conceivably suppressed.
\end{abstract}

Keywords: Active jet control, cylinder flow, jet momentum coefficient, aerodynamic forces; wake vortex evolution

\section{Introduction}

Structures with a cylindrical section are prevalent in engineering applications, examples include long stay cables of cable-stayed bridges, transmission lines, cooling towers, boat masts, chimney stacks and some modern high-rise buildings. When these cylindrical structures are exposed to wind, the surrounding airflow experiences a flow separation and leads to a periodic vortex street downstream in the near wake. This alternating process of vortex shedding generates highly unsteady aerodynamic forces acting onto the cylinder

\footnotetext{
* Corresponding author

Email address: cwl_80@hit.edu.cn (Wenli Chen)
} 
(Brika and Laneville (1993)). If the frequency of the periodic vortex shedding matches some-order modal frequency of the structure, a frequency-resonance will develop. In consequence, a fluid-structure synchronization called lock-in may lead to vortex induced vibrations (VIVs) of the structures. One typical case is the frequently observed VIVs of stay cables of cable-stayed bridges in the subcritical Reynolds number range (see Zuo and Jones (2010) for instance).

Most of the flow induced vibrations of structures are undesired. For example, the wind induced cable vibrations may lead to the breakdown of protections against corrosion and impair the cable-deck connections (Pacheco and Fujino (1993)). Therefore, various counter-measures have been proposed and developed to suppress the wind induced vibrations of structures. Since the alternating vortex shedding is the main cause of wind induced vibrations, a well established philosophy is to alleviate the vortex shedding from cylindrical structures thus to suppress the fluctuating lift forces. A great quantity of simple control schemes are introduced to control the periodic vortex shedding from a circular cylinder so as to decrease the drag and lift forces. Typical examples are splitter plates, guiding vanes, surface protrusions, base-bleed, as reviewed previously in Zdravkovich (1981). Recently, a passive jet flow control scheme was developed by Chen et al. (2015). Measurement results of flow structures demonstrate that the mechanism of passive jet control is to cause passive jets into the wake of cylinder and convert the vortex shedding mode from antisymmetric to symmetric. As a result, the drag is reduced and lift fluctuations suppressed. Bouak and Lemay (1998) presented a control by placing a smaller cylinder in the cylinder wake and interacting with the upstream main cylinder. The results show that at a stagger angle of $45^{\circ}$, the lift fluctuation of the upstream main cylinder is reduced by $41 \%$. Zhou et al. (2015) studied the flow around a cylindrical model with dimpled surface. The experiment results indicate that the dimpled surface contributes to a drag reduction of $10 \%$ and affects the vortex-shedding strength in the near wake of the cylinder. Oruç et al. (2016) conducted PIV measurements on flow around a cylindrical test model modified with drop-shaped mesh. They found that the vortex formation length is longer, the turbulence kinetic energy and normalized Reynolds shear stress contours are conspicuously weaker than the bare cylinder without control. Durhasan et al. (2016) used a perforated fairing coating on a circular cylinder to realize vortex street suppression. They observed that the wake formation region of the modified cylindrical model is elongated notably and the strength of shear flows are decreased compared to the natural case.

The schemes discussed above usually fall into a category of passive flow control, since they need no external energy to maintain the control process once implemented. Another kind of control schemes is active flow control. In a case of active control, continuous energy input is required to maintain the control process. Classical active control schemes are steady suction (Chen et al. (2013) and Chen et al. (2014)), synthetic jets (Feng et al. (2010), Feng and Wang (2010), Feng and Wang (2012)) and plasma actuators (Benard and Moreau (2013)). A steady suction flow control method was employed by Chen et al. (2013) to alleviate the periodic vortex shedding in the wake of a vibrating cylinder, thereby decrease the amplitude of its vortex induced vibration. The wind tunnel tests reveal that the steady suction flow control shows good control 
effectiveness by dramatically reducing the vibration amplitudes of the cylinder in the lock-in range. Followed by vibrational tests, the effects of active suction on manipulation of the wake vortex-shedding and suppression of the unsteady lift forces are investigated in detail by Chen et al. (2014). In addition, Feng et al. (2010), Feng and Wang (2010) and Feng and Wang (2012)) performed serial experiments to find that the synthetic jets implemented at the rear stagnation points of a cylindrical model act as external perturbations on the unsteady and periodic wake flows and lead to a notable modification to the alternating vortex-shedding process. Dong et al. (2008) introduced a control concept characterized by combined windward suction and leeward blowing (WSLB). This concept is investigated experimentally by Gao et al. (2018), and the test results show that the periodic vortex shedding in the cylinder wake is attenuated due to the action of WSLB control.

In the current study, we focus on the effects of a crucial control-parameter, i.e. the equivalent jet momentum coefficient $C_{\mu}$, on the active control of cylinder flow, including effects on the aerodynamic forces, wake flow evolutions and spanwise flow structures. This paper is organized as follows. Details of the cylindrical model with active jet control, the experiment set-up and the experimental campaign are given in Sec. 2, the test results are presented and analyzed in Sec. 3 and some concluding remarks are drawn in Sec. 4 .

\section{Experimental set-up}

The experimental campaign is performed in a low-turbulence wind tunnel (SMC-WT2), the Joint Laboratory of Wind Tunnel and Wave Flume (WTWF), Harbin Institute of Technology, China. This closed-circuit wind tunnel has a test chamber of $800 \mathrm{~mm}$ (width) $\times 1200 \mathrm{~mm}$ (height) $\times 3000 \mathrm{~mm}$ (length). Tunnel walls of the test chamber are designed to be transparent in order to facilitate flow diagnostics with optical techniques. Honeycombs and mesh structures are mounted ahead of a contraction section to produce uniform wind entering the test chamber. By using a Cobra Probe, the turbulence intensity level inside the test chamber we used in the current study is measured to be less than $0.40 \%$.

\subsection{Cylindrical model with active jets in the wake}

The cylindrical test model is made of a Polymethyl methacrylate (PMMA) tube with a smooth surface. The outer diameter of the cylindrical model (D) is $100 \mathrm{~mm}$, thus the solid blockage in the present experiment is $8.33 \%$. The horizontal test model, as shown in Fig.11, is fixed to the end plates and perpendicular to the incoming wind. The end plates are firmly locked onto the section walls. The test model has a total spanwise length (L) of $800 \mathrm{~mm}$ to adapt the chamber width. Four jet nozzles, with an inner diameter of 10 $\mathrm{mm}$, are manufactured at the leeward stagnations of the cylinder so the active jet flows will be evacuated to the cylinder wake along the same direction of the incoming airflow. The spanwise spacing between two neighboring jet nozzles, $\mathrm{L}_{0}$, is $\mathrm{L} / 8$, as shown in Fig.11. The jet nozzles are made with 3D printing technique to minimize the mechanical errors during manufacturing process. For every test case, the wind speed entering into the test chamber $U_{0}$ is fixed at $5.0 \mathrm{~m} \cdot \mathrm{s}^{-1}$, resulting in a Reynolds number, given the model diameter, 
viscosity of air, is $\operatorname{Re}=3.33 \times 10^{4}$. It is noted that $O\left(10^{4}\right)$ is a typical Re level for stay cables of cable-stayed bridges undergoing VIVs.
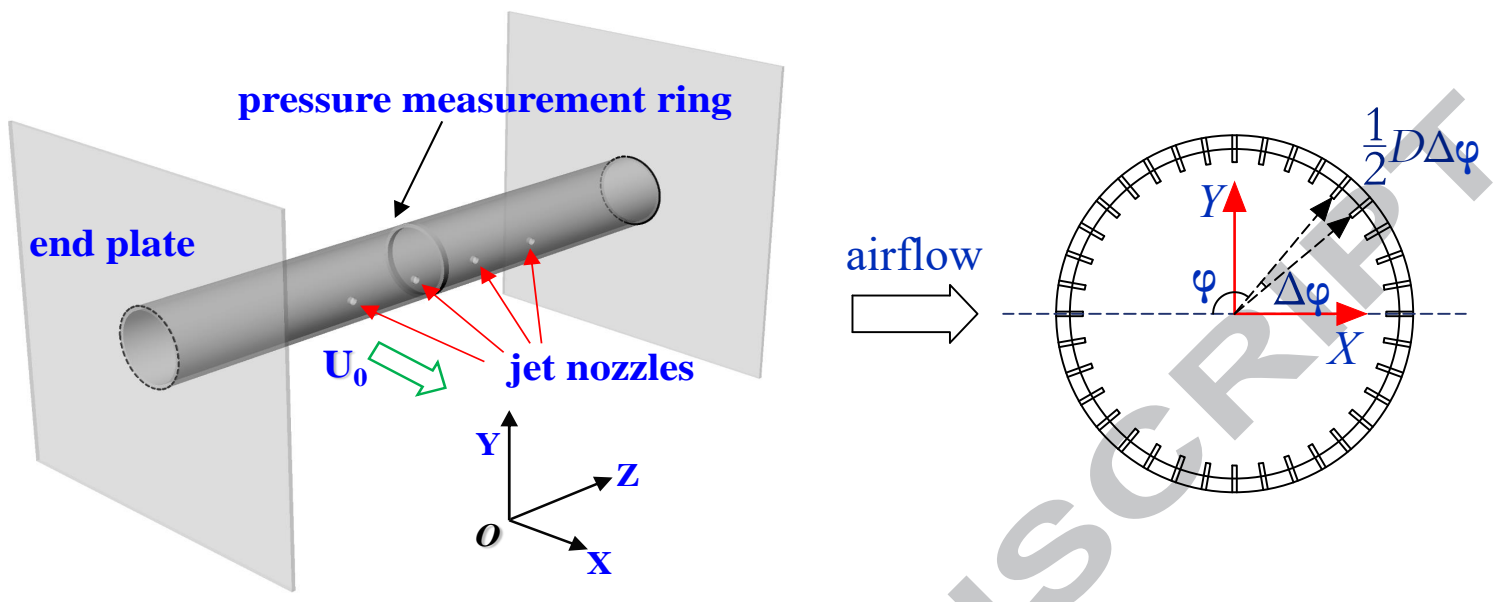

Figure 1: Schematics of the cylindrical test model (left) and the pressure measurement plane in the mid-span (right). Origin of the XYZ coordinate system is at the section center of the cylindrical slice in the mid-span.

In the present wind tunnel tests, a flow meter (Omega FMA-2613A) is employed to control the rate of jet flow from the nozzles. The jet rate $(Q)$ increases from $0 \mathrm{~L} \cdot \mathrm{min}^{-1}$ to $105 \mathrm{~L} \cdot \mathrm{min}^{-1}$ with a regular step of $7.5 \mathrm{~L} \cdot \mathrm{min}^{-1}$. It should be noted that the baseline case of $Q=0$ is also studied in the present study to provide reference values and vortex shedding patterns for comparison. When $Q$ is nonzero, we refer to it as a controlled case hereinafter. It is also helpful to introduce the concept of equivalent jet momentum coefficient $C_{\mu}$ in the present study. This dimensionless parameter is defined as the ratio of the jet momentum flux to the incoming momentum flux of freestream, i.e. $C_{\mu}=\left(\bar{U}_{j} / U_{0}\right)^{2}\left(S_{j} / D L_{0}\right)$. Here, $\bar{U}_{j}$ is the mean jet velocity from the nozzles, $S_{j}$ is the area of the jet nozzle at each jet section, and $\mathrm{L}_{0}=\mathrm{L} / 8$ is the interval between two neighboring jet nozzles. The jet-flow rate, jet velocity from the nozzles and its resultant jet momentum coefficient $C_{\mu}$ for the controlled cases are calculated and collected in Table. 1 .

Table 1: Jet flow rates and their corresponding jet momentum coefficients of different test cases in the present study

\begin{tabular}{c|c|c|c|c|c|c|c}
\hline \hline Jet flow rate $\left(\mathrm{L} \cdot \mathrm{min}^{-1}\right)$ & 7.5 & 15 & 22.5 & 30 & 37.5 & 45 & 52.5 \\
\hline Jet flow velocity $\left(\mathrm{m} \cdot \mathrm{s}^{-1}\right)$ & 1.5915 & 3.1830 & 4.7745 & 6.3660 & 7.9575 & 9.5490 & 11.1405 \\
\hline Jet momentum coefficient $\left(C_{\mu}\right)$ & 0.0008 & 0.0032 & 0.0072 & 0.0127 & 0.0199 & 0.0287 & 0.0390 \\
\hline \hline Jet flow rate $\left(\mathrm{L} \cdot \mathrm{min}^{-1}\right)$ & 60 & 67.5 & 75 & 82.5 & 90 & 97.5 & 105 \\
\hline Jet flow velocity $\left(\mathrm{m} \cdot \mathrm{s}^{-1}\right)$ & 12.732 & 14.3235 & 15.915 & 17.5065 & 19.098 & 20.6895 & 22.281 \\
\hline Jet momentum coefficient $\left(C_{\mu}\right)$ & 0.0509 & 0.0645 & 0.0796 & 0.0963 & 0.1146 & 0.1345 & 0.1560 \\
\hline \hline
\end{tabular}

\subsection{Measurement of surface pressure}

In the mid-span of the cylinder, a sectional pressure measurement ring is placed to measure the distributions of wind pressure on the cylinder surface, as can be noted in the left panel Fig.1. The pressure 
measurement ring is also made by a 3D printer and its constitutive material is photosensitive resin. 36 pressure taps are arranged axisymmetrically on the measurement plane, as sketched in the right subplot of Fig.1. A surface pressure measurement system that collects three models (DSA3217, range: 10 inH ${ }_{2} 0$; accuracy: $\pm 0.20 \%$ of full scale) is connected to the pressure taps in order to record the instantaneous surface-pressure distributions on the mid-span surface of the cylinder. The taps in the mid-span section and pressure scanivalves are connected via 36 Polyvinyl chloride (PVC) tubes. These PVC tubes have an uniform length of 500 $\mathrm{mm}$ and an inner diameter of $0.9 \mathrm{~mm}$. In the present study, the acquisition and recording for instantaneous surface pressure measurement last for $32 \mathrm{~s}$ for each test case and the sampling frequency is fixed at $312.5 \mathrm{~Hz}$, as in Gao et al. $2017 \mathrm{~b}$.

After pressure measurements, the time-variant aerodynamic drag and lift forces can be estimated by a standard integration process described in Chen et al. (2014). The instantaneous coefficients of the drag and lift forces, $C_{d, t}$ and $C_{l, t}$ can be estimated by:

$$
\begin{aligned}
C_{d, t} & =\frac{1}{2} \sum C_{p i} \Delta \varphi_{i} \cdot \cos \left(\varphi_{i}\right) \\
C_{l, t} & =\frac{1}{2} \sum C_{p i} \Delta \varphi_{i} \cdot \sin \left(\varphi_{i}\right)
\end{aligned}
$$

where $C_{p i}=2\left(p_{i}-p_{\infty}\right) / \rho U_{0}^{2}$ is the surface pressure coefficient, $p_{i}$ is the pressure acting on the test model and $p_{\infty}$ is the static pressure of incoming wind. Both the time-variant $p_{i}$ and $p_{\infty}$ are obtained by the pressure measurement system. $\varphi_{i}$ is the azimuthal angle that the $i$-th pressure tap locates, and $\Delta \varphi_{i}=10^{\circ}$ is the increment between two pressure taps, as shown in Fig.1. After obtaining the time-histories of the aerodynamic coefficients of the cylinder, counting the mean value of drag coefficients (denoted by $C_{D}$ ) and root mean square (RMS) value of lift coefficients (denoted by $C_{L}^{\prime}$ ) is helpful to assess the control effects of active wake-jet control for different jet momentum coefficients.

\subsection{PIV measurements}

We employ a particle image velocimetry (PIV) system in the current study to measure the flow characteristics around the controlled and bare cylinders. Figure 2 illustrates the experimental set-up for the two-dimensional PIV measurements. Small oil droplets of $\sim 1.0 \mu \mathrm{m}$ in size are produced to seed the flow. Illumination is provided by a double-pulsed Nd:YAG laser with a pulse energy of $425 \mathrm{~mJ} / \mathrm{pulse}$ at a wavelength of $532 \mathrm{~nm}$. The laser sheet is manipulated to be about $\sim 1.0 \mathrm{~mm}$ in thickness to illuminate the target sections. A high-resolution digital camera (PCO1600) is used for PIV image acquisition. The CCD camera and double-pulsed Nd:YAG lasers are communicated to a workstation via a Digital Delay Generator, thus the timing of the laser illumination and the image acquisition can be precisely controlled. In the PIV measurements, the repetition frequency of laser generator and PIV image acquisition is remained at $8 \mathrm{~Hz}$.

Two transverse planes, i.e. the mid-span plane $Z=0$ (coordinates are defined in Fig.1), one jet plane $Z / D=0.5$, and one horizontal plane $Y=0$ are selected as the target planes of PIV measurements (as sketched in Fig.2) to get a better understanding of three-dimensional characteristics of the cylinder flow modified with 

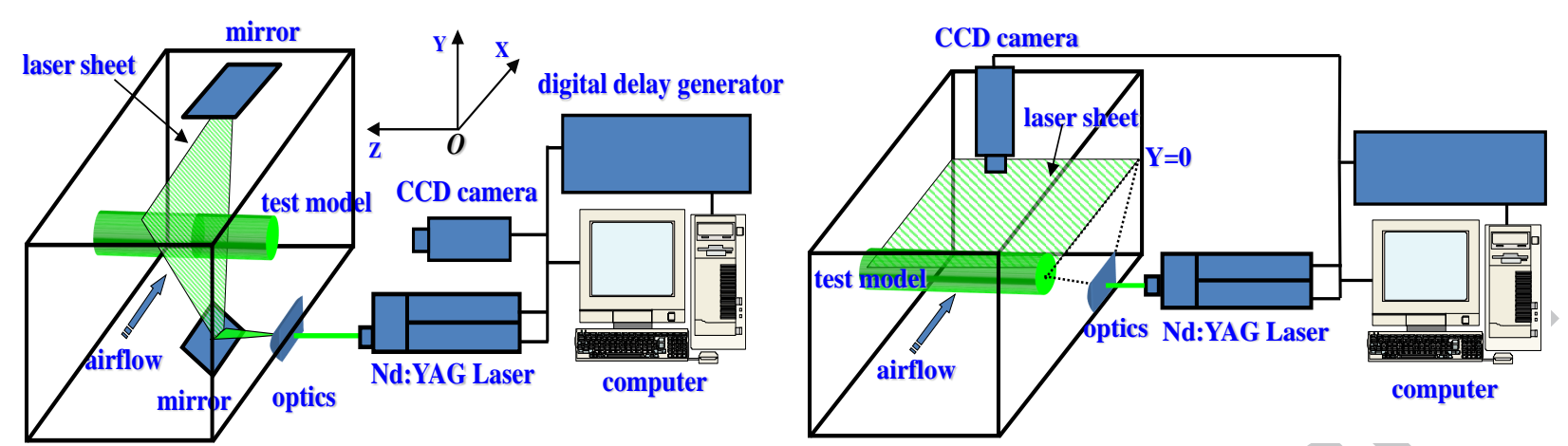

Figure 2: Experimental schematics for PIV measurement set-ups. Two streamwise target-planes of $Z=0$ and $Z / D=0.5$ (left) and a spanwise target-plane of $Y=0$ (right)

active jets. For every test case, the PIV system records more than 400 pairs of instantaneous images in order to arrive at a good convergence of measurement. In the image processing, a cross-correlation algorithm with an interrogation window size of $32 \times 32$ pixels and an effective overlap of $50 \%$ of the interrogation windows is applied to derive instantaneous flow vectors. If we take PIV measurements of $Z$-plane as an example, the velocity $(\boldsymbol{u}, \boldsymbol{v})$ and spanwise vorticity $\left(\boldsymbol{\Omega}_{\boldsymbol{z}}\right)$ can be readily determined by definition. Besides, the time-averaged flow characteristics, such as mean flow velocity $(\overline{\boldsymbol{u}}, \overline{\boldsymbol{v}})$, turbulence kinetic energy $(\mathrm{TKE}=$

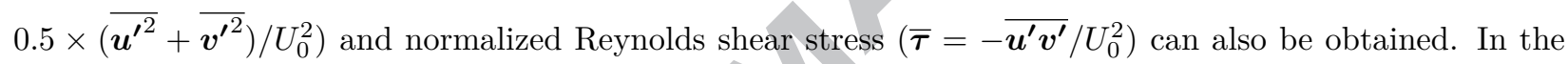
current PIV measurements, the uncertainty is estimated to be within $2 \%$ for the instantaneous flow vectors, whereas that of the time-averaged flow quantities is estimated to be less than 5\% (algorithm according to Park et al. (2008)).

\section{Experiment results}

\subsection{Results of aerodynamic forces}

Figure 3 presents the mean and fluctuating distributions of the surface-pressure for different jet momentum coefficients $C_{\mu}$. A symmetric region with negative pressure distributions is witnessed to develop at the leeward surface of the cylindrical test model without control. This is due to flow separation and it will lead to a recirculation region in the cylinder wake, as we shall see in section 3.2. With the implementation of active wake-jets, the regions of negative pressure distributions are generally lifted compared to the baseline case. It is noted that increasing the base pressure on the leeward surface of the cylinder contributes to a drag reduction. Besides, the base pressure distributions experience a gradual enhancement as the jet momentum coefficients increases to 0.0796 . This favorable effects will result in a gradually enhanced drag reduction. When $C_{\mu}$ shifts to 0.1146 , the pressure distributions experiences no significant change, indicating a relatively steady drag coefficient $C_{D}$. However, when $C_{\mu}$ continues to increase, the pressure distributions on the leeward surface of the cylindrical test model are decreased. In this case, $C_{D}$ will experience a upward tend and the drag reduction effect is weakened. It can also be noticed that the separation points of controlled cylinders are a little bit earlier than the bare cylinder. As will be revealed by PIV measurement results, the active-jet-flow 

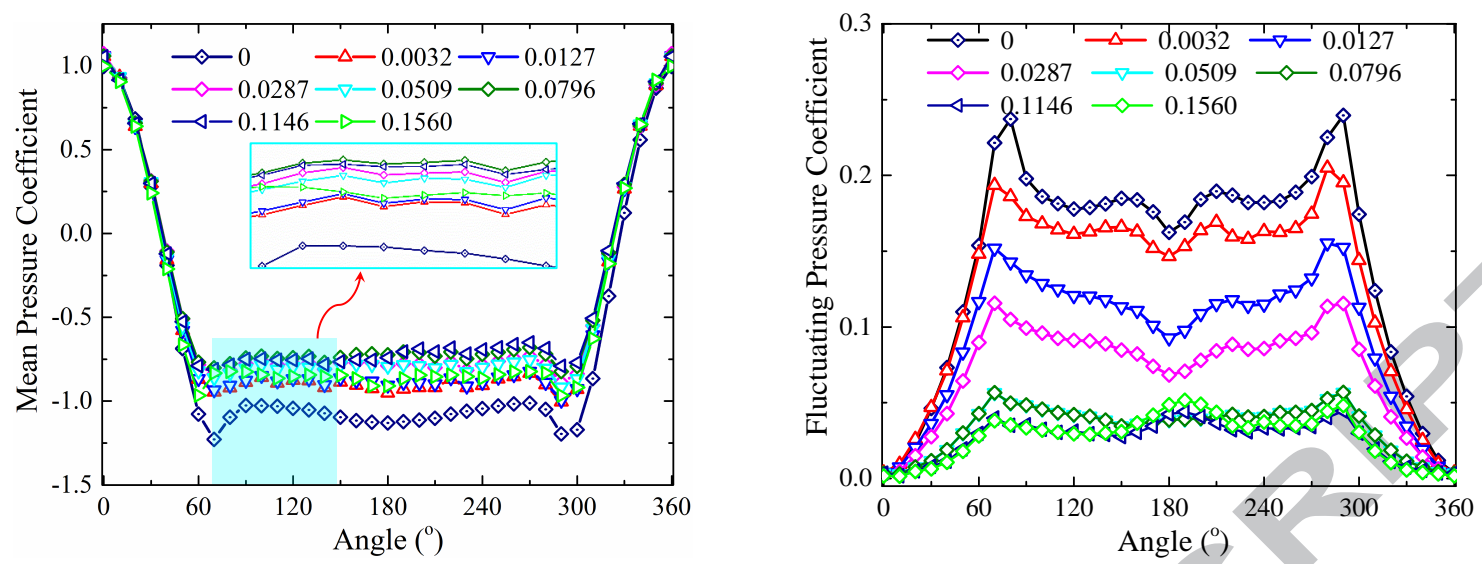

Figure 3: (Color online) Surface pressure distributions around the cylinder model with different $C_{\mu}$

into the wake interacts with shear flows from both sides of the cylinder. Accordingly, pressure distributions on the leeward surface are higher than the baseline case and the flow separation happens earlier. This pressure gradient further stabilizes the wake, thus to suppress the mean drag and alleviate the lift fluctuations. The root-mean-square (RMS) pressure coefficients of the controlled cylinders are also witnessed to be quite smaller than the baseline case, especially on the leeward surface of the cylindrical test model, as shown in Fig. 3. This implies a distinct suppression on the unsteady dynamic wind loads acting on the cylinder model. Unlike the mean pressure distributions, the RMS pressure distributions are regularly decreased to a certain level as $C_{\mu}$ goes up to 0.1146. In general, the pressure distributions in Fig. 3 reveal that a higher $C_{\mu}$ value contributes to a better control effectiveness in unsteady lift but not necessarily a better drag reduction.

Figure. 4 and Fig. 5 illustrate the time-variant signals of the drag and lift force coefficients of the cylinder with different $C_{\mu}$. It is shown that both the lift and drag forces are quite unsteady with significant fluctuations for the bare cylinder with zero $C_{\mu}$. The fluctuation are closely associated with the unstable and unsteady wakes of the bare cylinder, as will be revealed by PIV measurement results. When the active control is implemented, the fluctuations of lift forces are substantially decreased. Besides, for the controlled test cases with active jets, the drag coefficients of the controlled cylinders are found to be significantly smaller compared to the baseline case.

Figure 6 shows the non-dimensional frequency (Strouhal number, St $=f D / U_{0}$ ) of the time-variant histories of lift coefficients for different cases obtained by using a fast Fourier transform (FFT) analysis. It is observed that the dominant frequency from the bare cylinder is $9.98 \mathrm{~Hz}$ and results in a Strouhal number $\left(S t_{0}\right)$ of 1.996. A dominant frequency identified from the power spectrum of lift suggests a regular and periodic vortex shedding process from the cylindrical test model. Furthermore, when the cylinder is controlled with the active jet flow of $C_{\mu}=0.0287$, the dominant frequency experiences a marginal increase to $10.53 \mathrm{~Hz}\left(S t_{1}=2.106\right)$, indicating an acceleration of vortex shedding. In addition, the energy amplitude that the dominant frequency concentrates is found to be greatly decreased in comparison with the conventional vortex shedding from the bare cylinder. This change reveals a substantial modification and attenuation to 

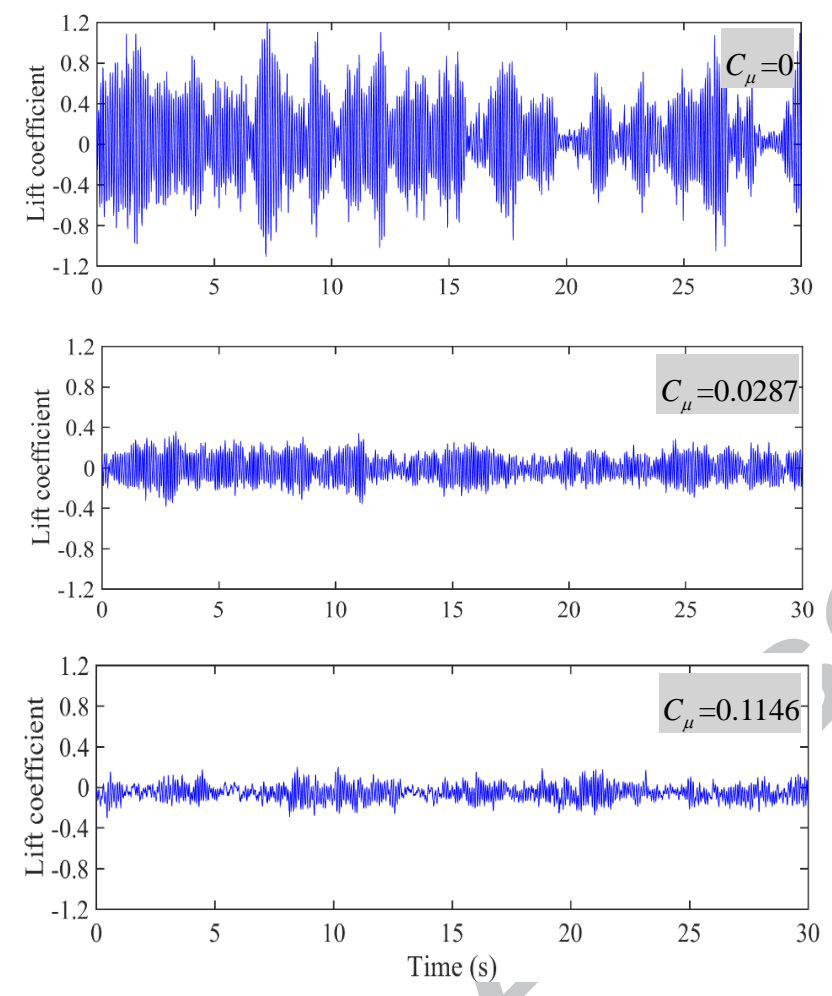

Figure 4: Time histories of the instantaneous lift coefficients acting on the cylindrical test model for three different $C_{\mu}$

the cylinder flow, especially the vortex shedding process. Moreover, when $C_{\mu}$ shifts to 0.1146 , the vortex shedding frequency is further increased to $10.55 \mathrm{~Hz}\left(S t_{1}=2.11\right)$, and its energy amplitude also decreased. It can be noted that with the increase of $C_{\mu}$, the control effectiveness of the active wake-jets to alleviate the unsteady and periodic vortex shedding is enhanced. These observations from pressure measurement results will be confirmed by PIV measurement results in the section that follows.

On the basis of the time sequences of the dynamic aerodynamic forces, the mean drag $\left(C_{D}\right)$ and RMS lift coefficients $\left(C_{L}^{\prime}\right)$ for different $C_{\mu}$ values (including zero $C_{\mu}$ value, i.e. the baseline case) are calculated and shown in Fig.7, to evaluate the active-jet control effectiveness more quantitatively. First, when $C_{\mu}$ is zero, $C_{D}$ and $C_{L}^{\prime}$ are calculated to be 0.36 and 1.12 respectively. After that, both $C_{D}$ and $C_{L}^{\prime}$ experience a downward trend as $C_{\mu}$ increases. This suggests that the active jet technique can reduce the drag and lift forces effectively. When $C_{\mu}$ equals $0.0796,17.8 \%$ drag reduction and $73.6 \%$ lift suppression are simultaneously reached. As $C_{\mu}$ continues to go high to 0.0963 and $0.1146, C_{D}$ remains stable. However, when $C_{\mu}$ reaches 0.1345 and $0.1560, C_{D}$ is increasing, indicating a less effective control in drag reduction. On the other hand, when $C_{\mu}$ exceeds $0.0963, C_{L}^{\prime}$ experiences an insignificant reduction and the optimal control effectiveness of fluctuating lift is $81.2 \%$ at the maximum $C_{\mu}$ of 0.1560 . Meanwhile, the decreasing trend is significantly slowed down, because the excitation level is so high that a saturation is obtained. It should be noted that these four jet nozzles are arranged isolatedly at the rear stagnation points, thus the momentum flux they inject into the wake flow is less efficient in flow modification than uniformly distributed jets along the spanwise direction 

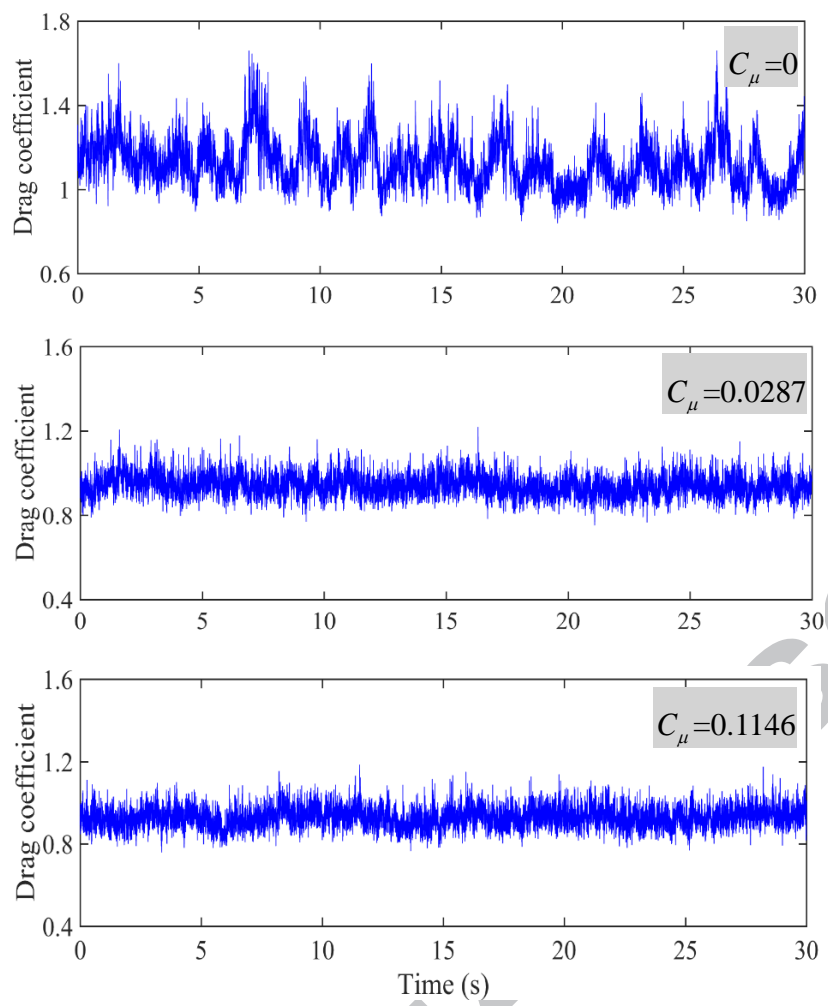

Figure 5: Time histories of the instantaneous drag coefficients acting on the cylindrical test model for three different $C_{\mu}$

(see Feng and Wang (2010) for instance). Furthermore, since the pressure taps are arranged in the plane with the longest distance from the jet nozzles, the active control scheme is expected to exhibit the least control effectiveness in the mid-span, i.e. the pressure measurement plane. Accordingly, the evaluation of control effectiveness on the basis of pressure distributions is believed to be on a conservative side and leads to an underestimated control effectiveness of mean-drag reduction and fluctuating-lift suppression of the circular cylinder.

\subsection{PIV measurement results}

In the post processing of flow images obtained by PIV system, a snapshot proper orthogonal decomposition (POD) method (Sirovich (1987), Meyer et al. (2007)) is employed to quantify the flow characteristics of the streamwise and spanwise wakes of the cylinder so as to investigate the control mechanism of active wake-jets. The energy map of POD modes for different cases is plotted in Fig. \&. It is shown that the several leading modes occupy the most energy for all the test cases, since the first POD modes reflect the large-scale and dominant coherent-structures of the global flow field. In the present study, flow structures are reconstructed by employing the first six POD modes, as in Gao et al. (2017a).

Figure 9 shows the mean flow structures behind the mid-section of the cylinder with different $C_{\mu}$. For the baseline case without active jet control, i.e. $C_{\mu}=0$, the flow separations take place near the angles of $90^{\circ}$ and $270^{\circ}$. Because of the flow separation, a recirculation region will be formed in the near wake. 


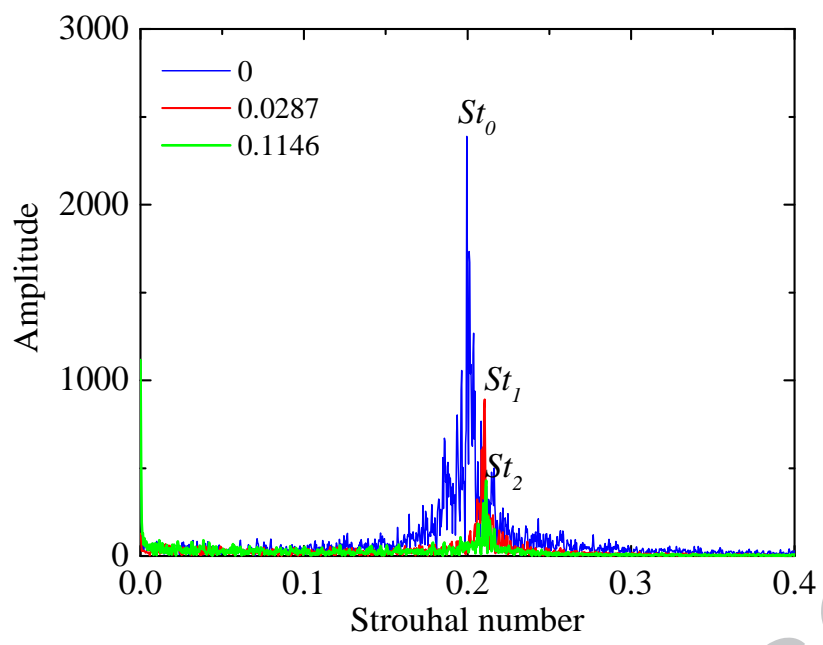

Figure 6: (Color online) Strouhal numbers for three different $C_{\mu}: S t_{0}=1.996 ; S t_{1}=2.106 ; S t_{2}=2.11$

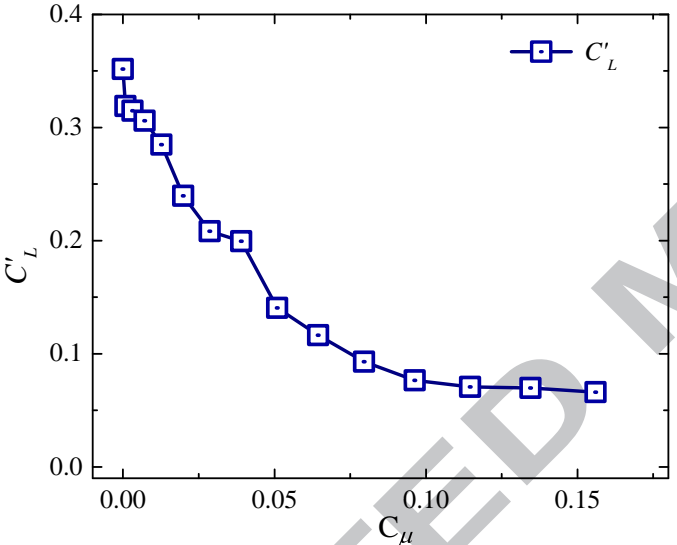

(a) RMS values of lift coefficient against $C_{\mu}$

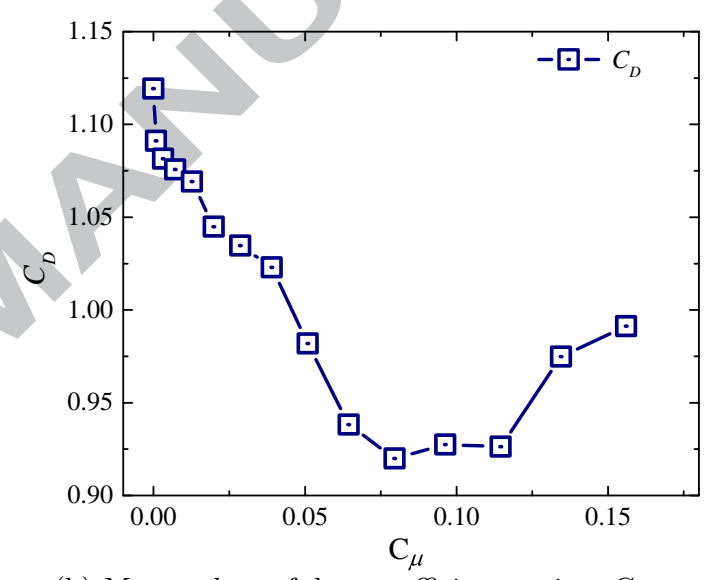

(b) Mean values of drag coefficient against $C_{\mu}$

Figure 7: Effects of $C_{\mu}$ on aerodynamic coefficients acting on the cylindrical test model

Besides, the turbulence kinetic energy (TKE) distributions in the wake of bare cylinder are witnessed with quite high amplitudes, especially along the vortex shedding paths. However, as $C_{\mu}$ increases, the overall TKE distributions in the wake are gradually suppressed, as shown in Fig.9. It should be noted that the TKE distributions are related to the turbulent vortex structures and are usually adopted as an indicator of the turbulence mixing of the wake flow and the unsteadiness and fluctuations of the surface pressures (Chen et al. (2014)). Consequently, a higher $C_{\mu}$ leads to a more stabilized wake and a less fluctuating amplitudes of lift forces, i.e. a smaller $C_{L}^{\prime}$, as presented in Fig.7. Another notable observation in Fig.9 is that the wake formation regions of controlled cylinders are elongated in comparison with the baseline case. This will be addressed in detail associated with spanwise flow structures.

To get an insight into the mechanism of active wake-jets, it is helpful to pay more attention to the jet section $(\mathrm{Z} / \mathrm{D}=0.5)$. Figure 10 presents the instantaneous vortical structures behind the wake-jet section for different test cases. As revealed by the instantaneous PIV measurement results, a pair of antisymmetric 


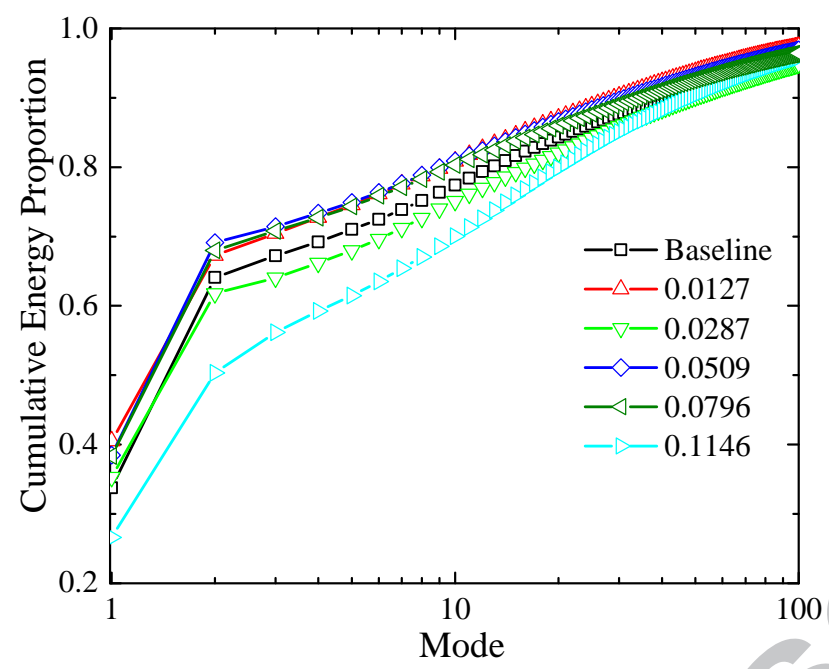

Figure 8: Cumulative energy proportion of the first 100 POD modes

vortical structures sheds periodically from the bare cylinder $\left(C_{\mu}=0\right)$ to form a Karman vortex street in the cylinder wake. This alternating and unsteady process from the solid-wall of cylinder will lead to dramatic pressure fluctuations on the cylinder model, especially on its leeward surface, as can be observed in Fig. 3 . Therefore, both lift and drag forces will fluctuate significantly and can possibly excite VIV if the cylinder is free to move. With the implementation of active jet of $C_{\mu}=0.0127$, a pair of symmetric and counter-rotating jet vortices are evacuated into the cylinder wake to detach the alternating separation flows generated at both sides of the cylinder. As a result of this dynamic competition process, the vortex formation region is elongated and shifted downstream. As $C_{\mu}$ increases, the jet vortices are gradually strengthened, and the competitions between the jet flow and the shear flows are gradually enhanced. When $C_{\mu}$ reaches a high level $\left(C_{\mu}=0.0796\right.$ or higher), two nearly symmetric arrays of vortices can be noticed in the near wake region, suggesting the wake pattern has nearly been converted into a symmetric mode. It is well accepted that the fluctuating lift of a cylinder is closely linked to the alternating vortex shedding. Therefore, a strengthened competition between the jet flow and the shear flows will alleviate the periodicity of the wake flow and result in a smaller $C_{L}^{\prime}$ (as plotted in Fig. 7). In addition, Liu and Feng (2015) also found that symmetric perturbations acting on the cylinder flow lead to a great change of the wake mode from the conventionally periodic one to a nearly symmetric one and could suppress effectively the lift fluctuations acting on the circular cylinder.

Derived by PIV measurement results, Fig.11 plots the mean flow topology behind the jet section for different natural and controlled cases. The jet flow into the wake interacts with the recirculating flow behind the cylindrical test model. In consequence, the recirculation region is enlarged and the near-wake width increased, in comparison with the bare cylinder $\left(C_{\mu}=0\right)$. Besides, even when $C_{\mu}$ is small, the highTKE region is shifted away from the cylinder wall, indicating a stabilized mode in the very near wake. Consequently, the fluctuation of the surface pressure and the resultant dynamic wind loads of the cylinder are decreased. Furthermore, as $C_{\mu}$ increases, the distributions of TKE in the cylinder wake are significantly 


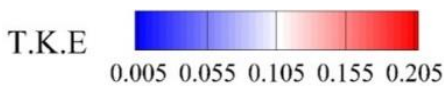
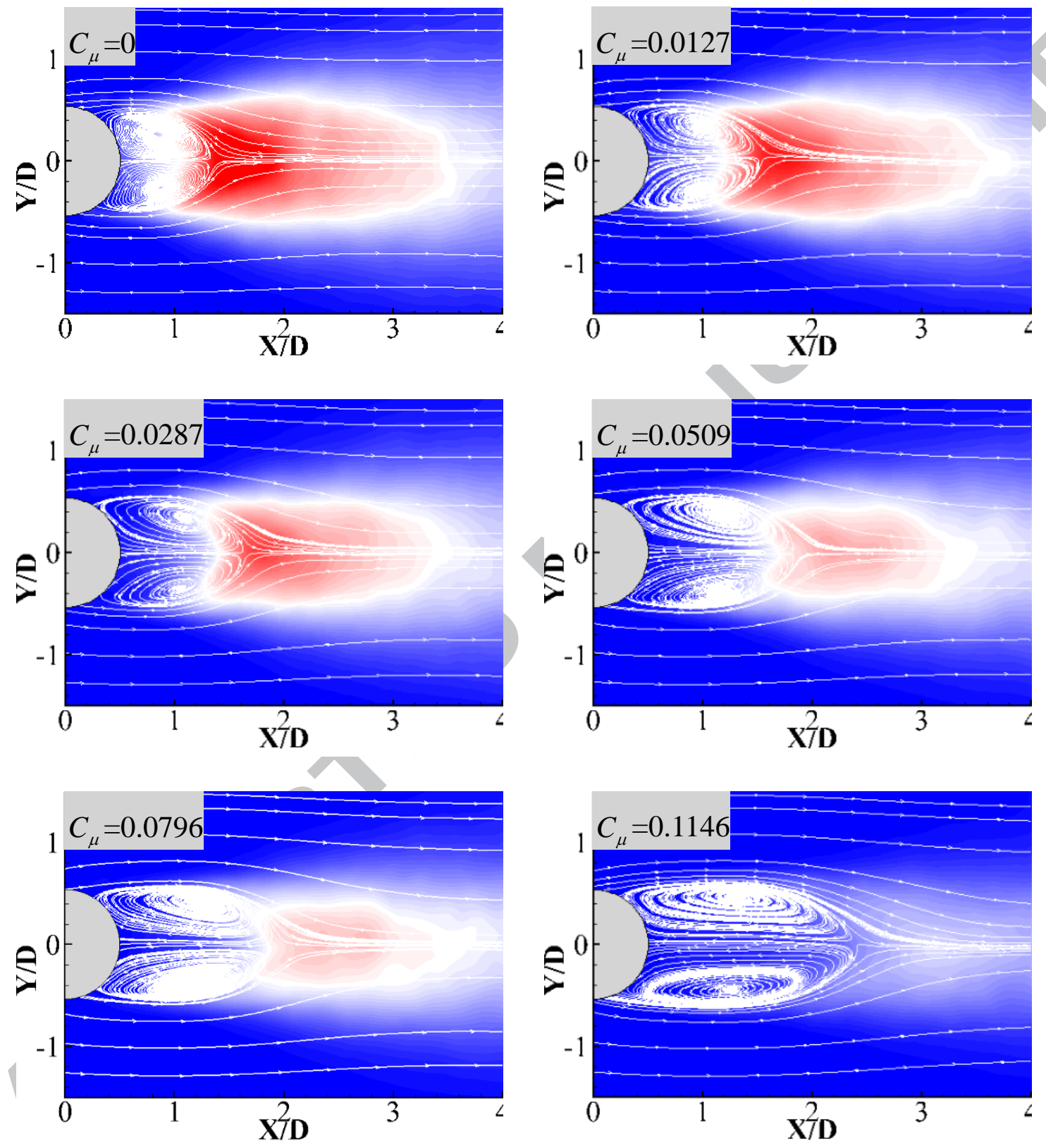

Figure 9: (Color online) The mean flow field measured by PIV system in the mid-span section of the cylindrical test model for different $C_{\mu}$ 


\section{ACCEPTED MANUSCRIPT}
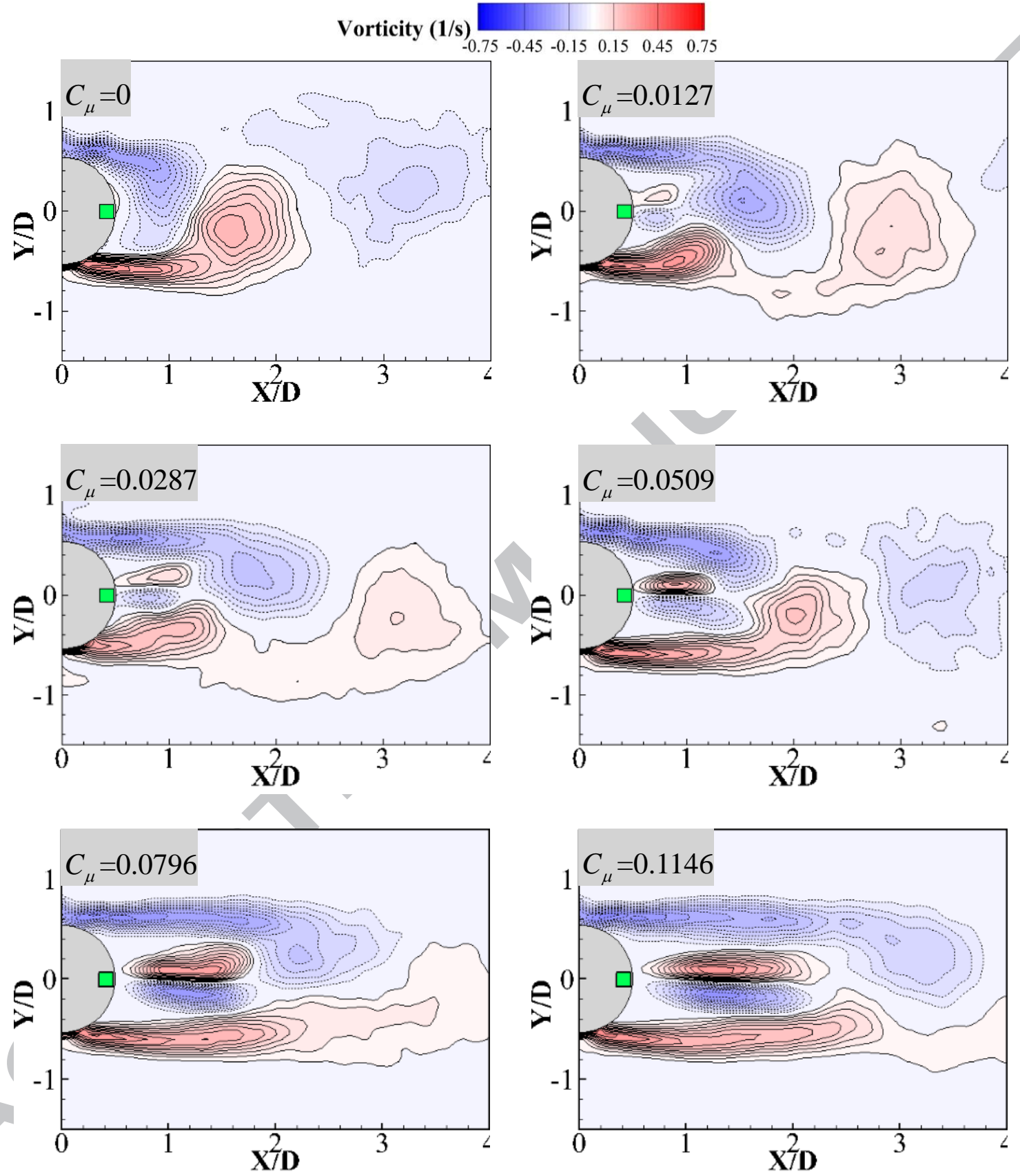

Figure 10: (Color online) The instantaneous flow strucutres measured by PIV system in the jet section of the cylindrical test model for different $C_{\mu}$ 
modified owing to the gradually enhanced active jets. The overall TKE levels in the wake flow become much smaller compared to the baseline case, as shown in Fig.11. When $C_{\mu}$ becomes 0.1146, TKE distributions in the entire wake are suppressed to a extremely low level, accompanying a better suppression in the fluctuating aerodynamic forces.

The mean recirculation region of the cylinder flow is characterized by the vortex formation length $\left(L_{v f}\right)$. In the present study, $L_{v f}$ is defined as the length between the cylinder center and the saddle point of timeaveraged streamtraces along the centerline of the test model. It is shown in Fig. 12 that the wake-jet flow control causes $L_{v f}$ to grow considerably longer, i.e. the vortex formation region is elongated to a further location in the wake. Besides, a lager $C_{\mu}$ is found to result in a larger recirculation region. This is due to wake-jet flow that prevents the interactions of shear layers from forming the alternating vortex shedding in the near wake. When $C_{\mu}$ is sufficiently large, two shear layers from two sides of the cylinder are detached and elongated to be nearly parallel, as shown in Fig.10.

According to the mean flow structures measured by PIV system, Fig.13 illustrates the time-averaged profiles of streamwise velocity at variant $\mathrm{X} / \mathrm{D}$ stations (where $\mathrm{X} / \mathrm{D}=0$ denotes the cylinder center) behind the cylindrical test model. In the first subplot of the velocity profiles, i.e. at $\mathrm{X} / \mathrm{D}=1.0$, peaks are readily witnessed near the jet nozzle $(\mathrm{Y} / \mathrm{D}=0)$, indicating a great modification to the wake flows near the cylinder wall. Generally, a stronger jet flow, denoted by a larger $C_{\mu}$, results in a more noticeable peak. As X/D shifts downstream to 1.5 , the peaks of cusp are weakened in comparison with the former case. This is due to the competition of the wake-jet flow and the backward flow in the recirculation region. When X/D moves farther downstream, this competition will decrease the jet flow velocity consistently and the jet cusp disappear gradually. As a result, the mean velocity profiles recover gradually with the increase of X/D. In addition, Fig. 14 presents the distributions of mean Reynolds stress, which is a significant parameter to characterize fluctuations of velocity in the wake. The distributions are nominally antisymmetric about the jet nozzle $(Y=0)$, which is due to the opposite directions of spanwise velocity induced by the counter-rotating vortex pairs. Besides, as X/D shifts from 1.0 to 1.5, the Reynolds stress increases. In this region, jet flow from the nozzle is developed and broadened, indicating that the turbulent velocity fluctuations are enhanced and so are the Reynolds stress distributions. After that, the Reynolds stress distributions experience a gradual decrease, because the wake flow gradually recover towards incoming airflow and its fluctuations both in streamwise and transverse directions are gradually weakened. It can also be noted that, at each X/D station, the Reynolds stress tends to diminish with the increase of $C_{\mu}$, suggesting that the stronger the wake-jet flow is, the more active the momentum exchange between the active jet and the surrounding airflow will be.

Figure 15 present different profiles of the fluctuating streamwise velocity. The figure shows that two peaks lying at the two sides of the centerline behind the bare cylinder, i.e. a double-cusp pattern. However, the streamwise velocity fluctuation profiles exhibit quadruple-cusp patterns in the near wake for the controlled cylinders. This is associated with the swirling patterns of vortices formed in the recirculation region. When $\mathrm{X} / \mathrm{D}$ moves farther downstream and gets rid of the backward flow in the recirculation region, the profiles 


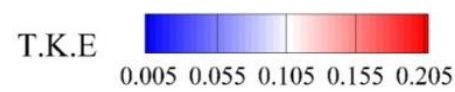
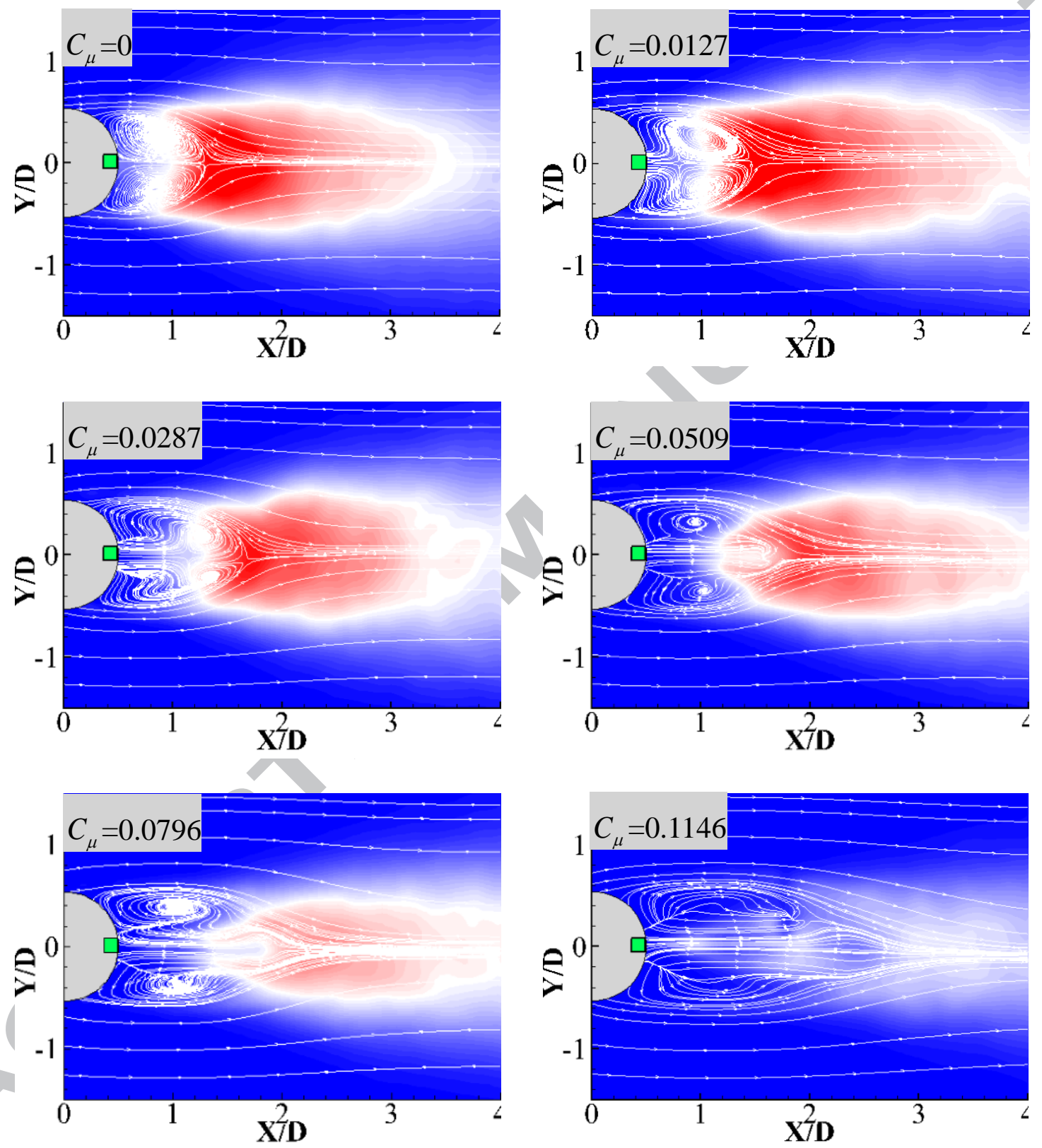

Figure 11: (Color online) The mean flow field measured by PIV system in the jet section of the cylindrical test model for different $C_{\mu}$ 


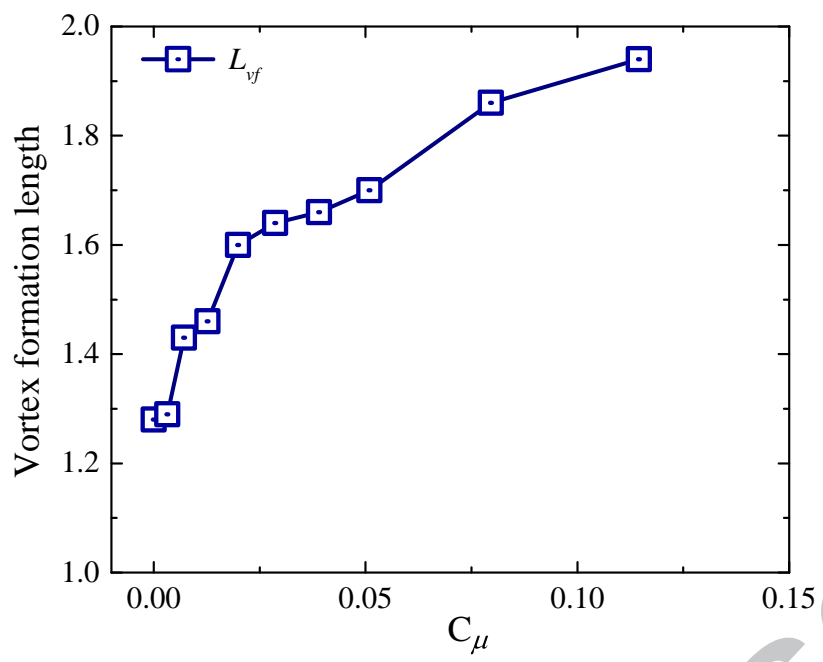

Figure 12: The time-averaged vortex formation length behind the cylindrical test model for different $C_{\mu}$

all switch to be double-cusp. The distributions of fluctuating transverse velocity are shown in Fig. 16. The transverse fluctuation of velocity exhibits similarity to the Reynolds stress distributions. It is known that the interaction between the shear-layers in the wake affects crucially the transverse velocity fluctuation. Therefore, the vortex interaction in the near wake of the controlled cylinder is alleviated and prevented to some extent, as shown in the instantaneous wake flows discussed above.

We note different wake flow patterns behind the mid-span and jet sections (Figs. 9 and 11) due to the three-dimensional nature of the cylinder flow with active jet. The spanwise flow structures measured at the jet plane $(\mathrm{Y} / \mathrm{D}=0)$ are illustrated in Fig.17. For the baseline case, there is a velocity region where the backflow obviously forming a recirculation region behind the cylinder, as can also be noted in Fig.11. For the controlled cylinders, the jet vortex pairs with opposite rotations can be clearly identified for controlled cases. It is found that the instantaneous flow structures at $\mathrm{Y} / \mathrm{D}=0$ are nearly stable and symmetric about the jet nozzle. This observation is different from the wake vortices at the plane of $\mathrm{Z} / \mathrm{D}=0.5$. The latter ones are due to the interaction with the shear layers from upper and lower sides of the cylinder. In addition, the active jet flow are observed to affect a farther region along the streamwise direction and the recirculation regions are pushed further downstream with a larger $C_{\mu}$. This is consistent with above discussions.

\subsection{Discussions}

It is noted in Fig.9 that $C_{D}$ increases while $C_{L}^{\prime}$ trends to be stable as $C_{\mu}$ exceeds a turning point about 0.10. The trend in mean drag is similar to some previously reported results. For instance, Apelt et al. (1973) investigated the flow around a circular cylinder modified with a splitter plate and they observed that the mean drag of the cylinder with a short splitter plate $(\mathrm{L} / \mathrm{D}<1)$ would progressively decrease with the increase of splitter length $(\mathrm{L})$. However, as the splitter length exceeds a critical value $(\mathrm{L} / \mathrm{D}=1)$, the drag coefficient experiences an upward trend and the drag reduction effect is reduced, as illustrated in Fig.5 of Apelt et al. (1973). 

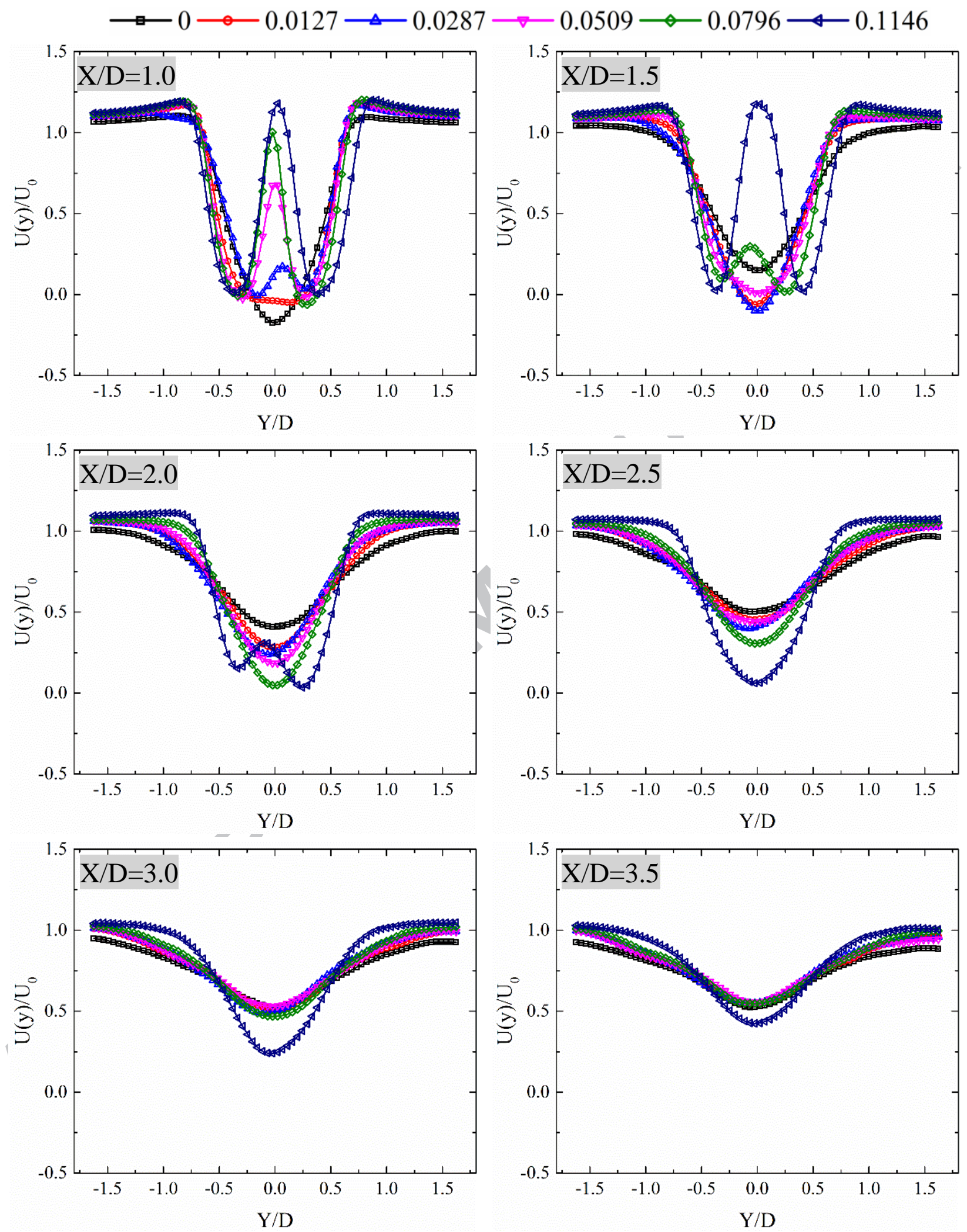

Figure 13: (Color online) Profiles of the mean streamwise velocity behind the cylindrical test model for different $C_{\mu}$ 

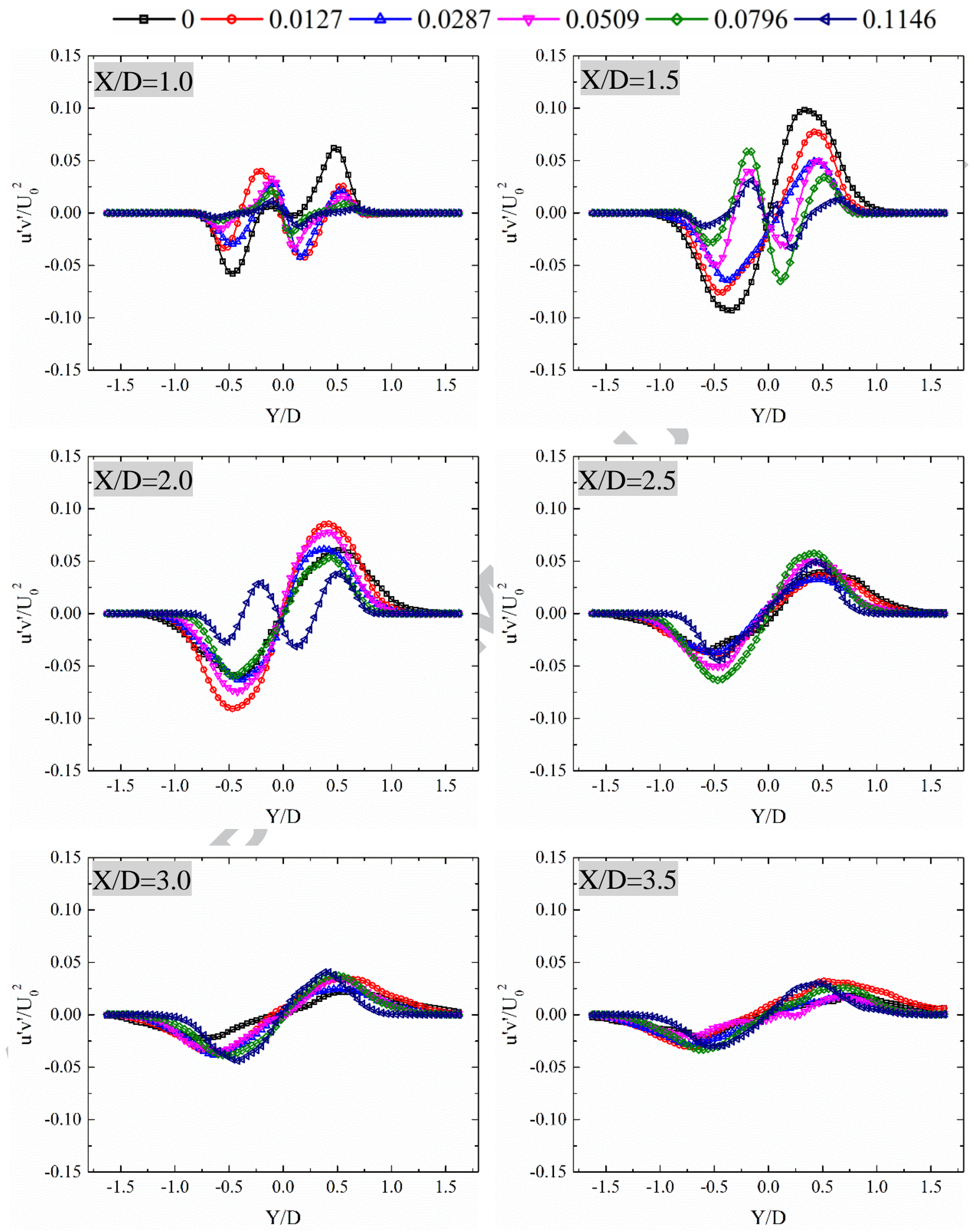

Figure 14: (Color online) Reynolds stress distributions behind the cylindrical test model for different $C_{\mu}$ 

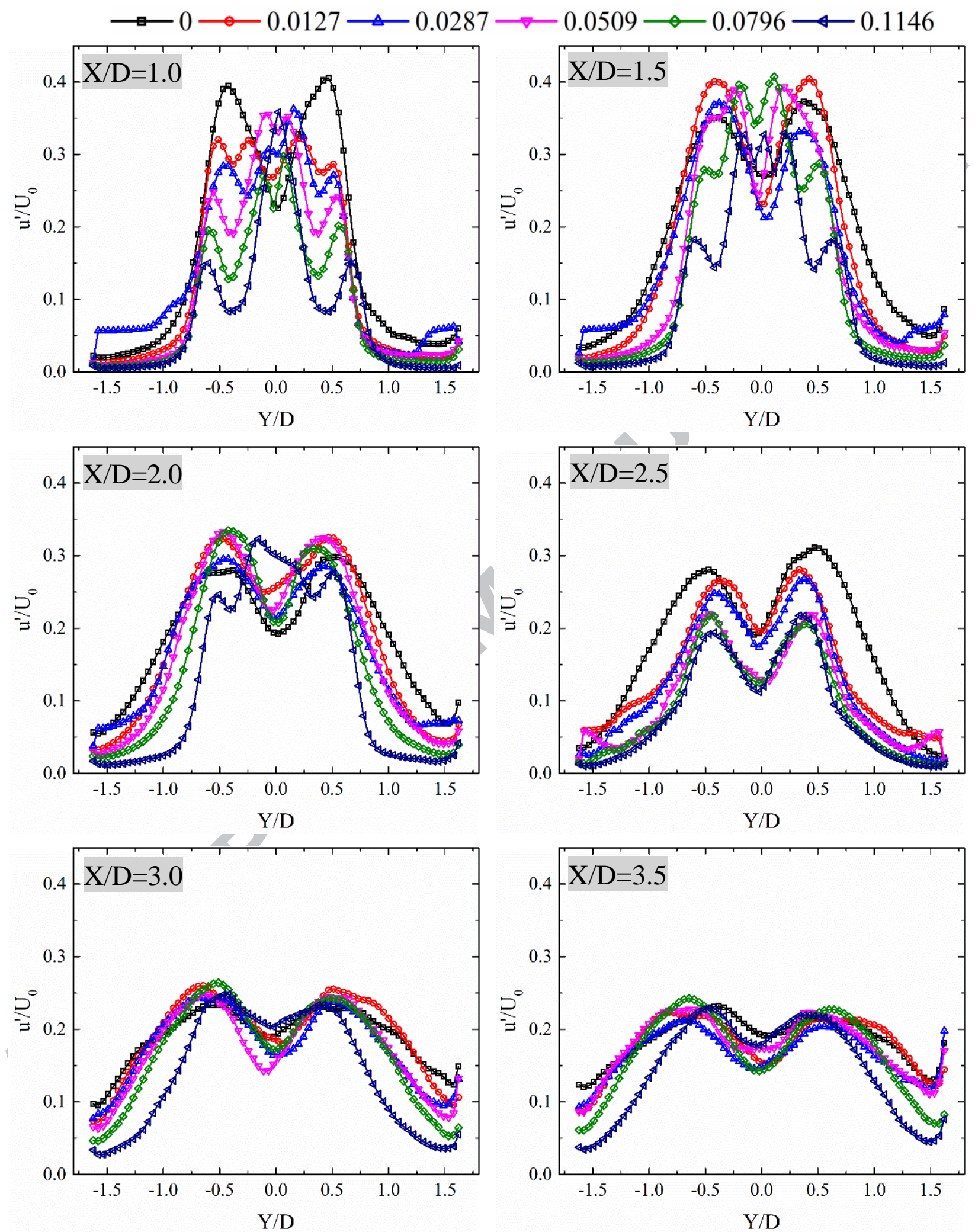

Figure 15: (Color online) Profiles of the fluctuating streamwise velocity behind the cylindrical test model for different $C_{\mu}$ 

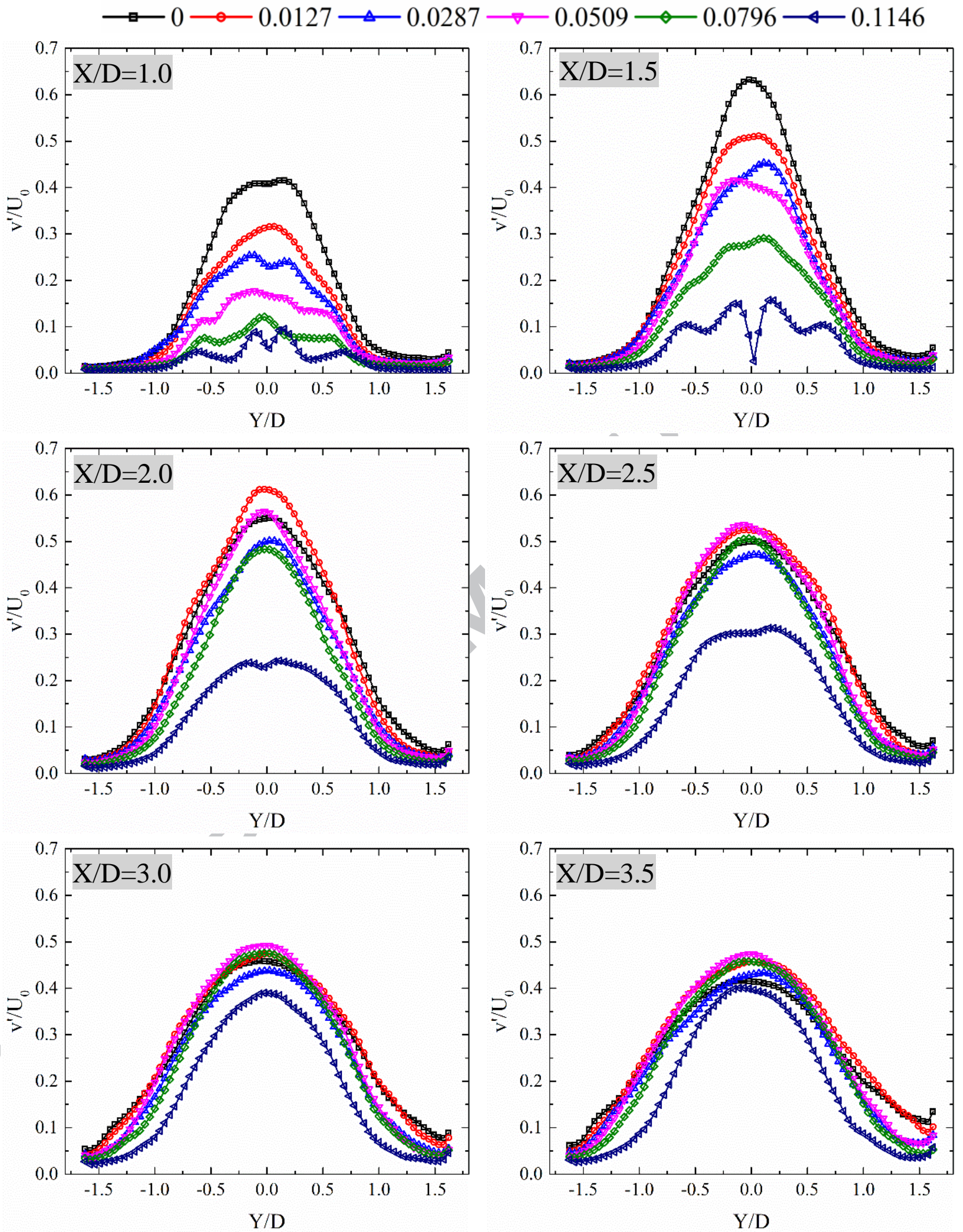

Figure 16: (Color online) Profiles of the fluctuating transverse velocity behind the cylindrical test model for different $C_{\mu}$ 


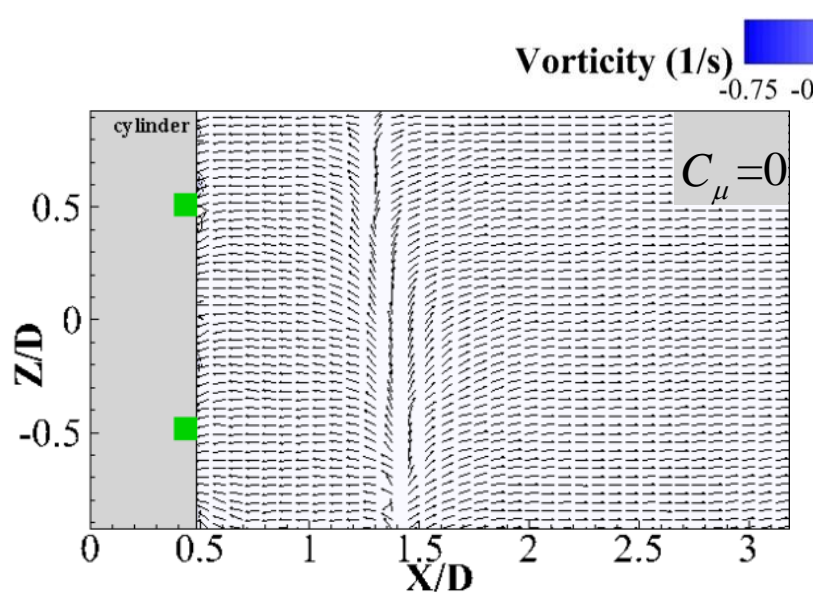

$\begin{array}{lllll}0.45 & -0.15 & 0.15 & 0.45 & 0.75\end{array}$
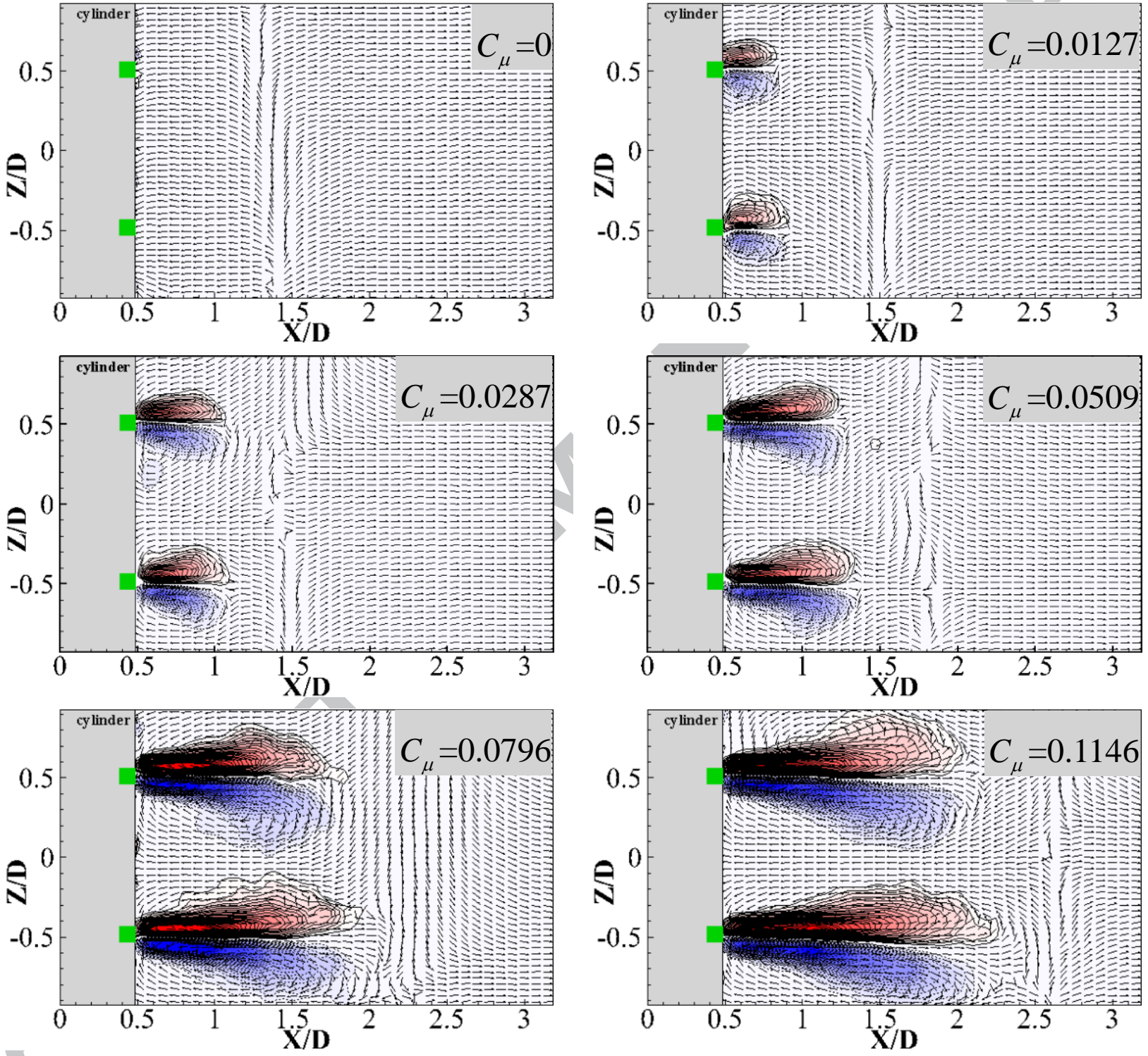

Figure 17: (Color online) The instantaneous flow structures in the plane of $\mathrm{Y}=0$ for different $C_{\mu}$ 


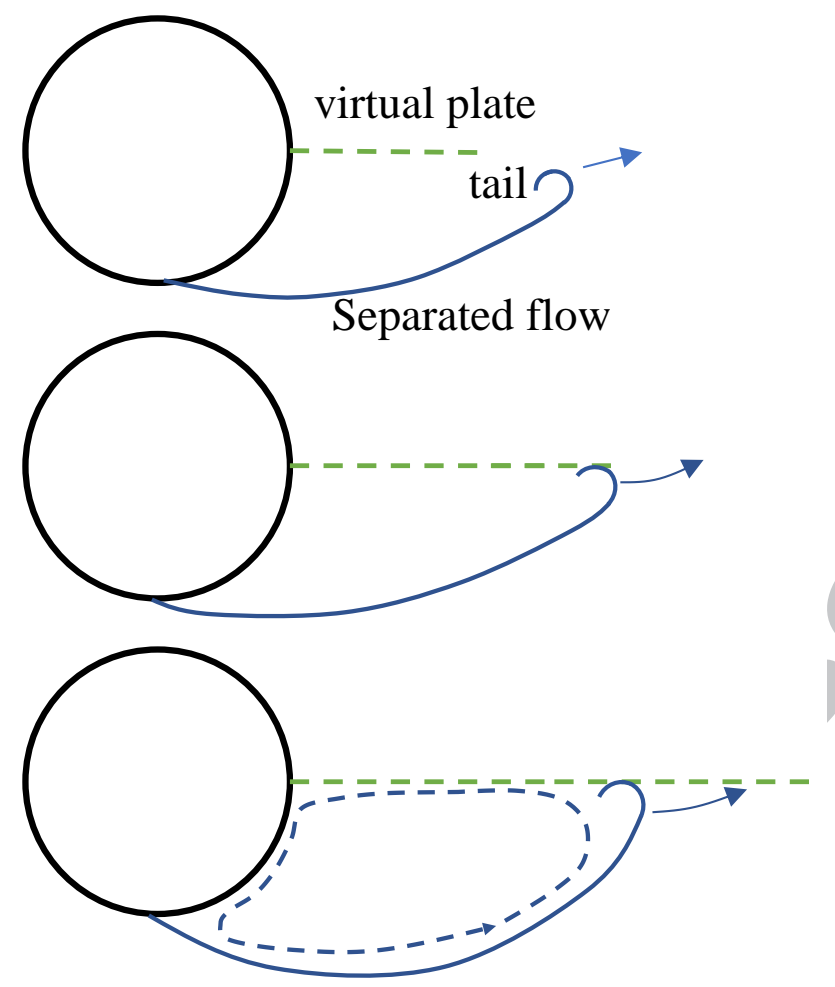

Figure 18: Schematics of the vortex formation behind a circular cylinder with virtual splitter-plate

Amitay et al. (1997) employed synthetic jets to modify the aerodynamic behaviors of a circular cylinder. They suggested that the recirculating regimes near the solid wall acted as a 'virtual surface' to push the streamlines outside. From a similar point of view, the effects of wake jets in the present study are actually to form a 'virtual plate' in the cylinder wake (as shown in Figs.11 and 17), so the aerodynamic behaviors of the controlled cylinder in the present study exhibit similar trends to the cylinder hinged with a solid wake-splitter. The schematics in Fig. 18 illustrates the saturation effects of 'virtual plate' on vortex formation process. When $C_{\mu}$ is small, the virtual plate is short (the upper subplot), and the plate prevents the interaction of separated flows. This prevention process is gradually enhanced with the increase of virtual plate $\left(C_{\mu}\right)$, so $C_{L}^{\prime}$ will witness a gradual decrease. The middle subplot of Fig.18 represents the turning point. In this case, the tails of shear flows touch at the trailing edge of the virtual plate. Furthermore, when $C_{\mu}$ continues to grow, the enlarged length of virtual plate will not further create significant change to the vortex formation in the wake (see the lower subplot). As a result, $C_{L}^{\prime}$ remains relatively stable.

\section{Conclusion}

In the current study, a wind tunnel investigation is conducted at a Reynolds number of $\operatorname{Re}=3.33 \times$ $10^{4}$ to investigate the effectiveness of active wake-jets (characterized by a dimensionless jet momentum coefficient $\left(C_{\mu}\right)$ ) to manipulate the vortex shedding patterns so as to decrease the fluctuating amplitudes of the aerodynamic coefficients. Both surface pressure and detailed flow measurements (PIV) are performed 
to study the flow characteristics of the cylindrical model with different $C_{\mu}$. The principal conclusions from this experimental work are as follows.

The active jet into the wake can suppress effectively the drag and lift forces. On the basis of pressure measurement results, the control effectiveness on fluctuations of lift coefficient meets a saturation when $C_{\mu}$ exceeds 0.0963. Compared to the bare cylinder, the RMS value of lift forces is estimated to be decreased up to $81.2 \%$ at the $C_{\mu}$ of 0.1560 . Besides, the best drag reduction (about 16-18\%) is achieved in the $C_{\mu}$ range of 0.0796-0.1146. As $C_{\mu}$ continues to go high, the control effect of drag reduction is slightly decreased. In addition, with the implementation of the active jet control, the vortex shedding frequency is minimally increased.

The PIV measurement results confirmed the observations from the pressure measurement. Compared to the baseline case, the recirculation regions behind the controlled cylinder are elongated and pushed downstream. Moreover, the TKE distributions in the wake flow behind the controlled cylinders are much smaller than the baseline case, indicating stabilized cylinder wakes. The instantaneous wake vortex structures reveal that these great modifications to the cylinder flow are due to the interaction of the jet flows with the shear flows from both sides of the cylinder. With sufficiently large $C_{\mu}$, the alternating shear layers are switched into two parallel arrays. To conclude, the mechanism of the active wake-jet control is to impose steady and symmetric perturbations into the unsteady and asymmetric flows in the cylinder wake, so as to alleviate the periodic vortex shedding and reduce the fluctuations of aerodynamic forces effectively.

\section{Acknowledgments}

We wish to acknowledge the supports awarded by the National Nature Science Foundation of China through Grants 51378153, 51578188, and 51722805, and the Fundamental Research Funds for the Central Universities (HITBRETIV. 201803 and HITBRETIII. 201512). D. G also acknowledges the financial support from China Scholarship Council (CSC 201606120189).

\section{References}

Amitay, M., Honohan, A., Trautman, M., Glezer, A., Amitay, M., Honohan, A., Trautman, M., Glezer, A., 1997. Modification of the aerodynamic characteristics of bluff bodies using fluidic actuators, in: 28th Fluid Dynamics Conference, p. 2004.

Apelt, C., West, G., Szewczyk, A.A., 1973. The effects of wake splitter plates on the flow past a circular cylinder in the range $104<\mathrm{r} 4$. Journal of Fluid Mechanics 61, 187-198.

Benard, N., Moreau, E., 2013. Response of a circular cylinder wake to a symmetric actuation by non-thermal plasma discharges. Experiments in fluids 54, 1467.

Bouak, F., Lemay, J., 1998. Passive control of the aerodynamic forces acting on a circular cylinder. Experimental Thermal and Fluid Science 16, 112-121. 
Brika, D., Laneville, A., 1993. Vortex-induced vibrations of a long flexible circular cylinder. Journal of Fluid Mechanics 250, 481-508.

Chen, W.L., Gao, D.L., Yuan, W.Y., Li, H., Hu, H., 2015. Passive jet control of flow around a circular cylinder. Experiments in Fluids 56, 201.

Chen, W.L., Li, H., Hu, H., 2014. An experimental study on a suction flow control method to reduce the unsteadiness of the wind loads acting on a circular cylinder. Experiments in fluids 55, 1707.

Chen, W.L., Xin, D.B., Xu, F., Li, H., Ou, J.P., Hu, H., 2013. Suppression of vortex-induced vibration of a circular cylinder using suction-based flow control. Journal of Fluids and Structures 42, 25-39.

Dong, S., Triantafyllou, G.S., Karniadakis, G.E., 2008. Elimination of vortex streets in bluff-body flows. Phys. Rev. Lett. 100, 204501.

Durhasan, T., Aksoy, M., Pinar, E., Ozkan, G., Akilli, H., Sahin, B., 2016. Vortex street suppression of a circular cylinder using perforated semi-circular fairing in shallow water. Experimental Thermal and Fluid Science 79, $101-110$.

Feng, L.H., Wang, J.J., 2010. Circular cylinder vortex-synchronization control with a synthetic jet positioned at the rear stagnation point. Journal of Fluid Mechanics 662, 232-259.

Feng, L.H., Wang, J.J., 2012. Synthetic jet control of separation in the flow over a circular cylinder. Experiments in fluids $53,467-480$.

Feng, L.H., Wang, J.J., Pan, C., 2010. Effect of novel synthetic jet on wake vortex shedding modes of a circular cylinder. Journal of Fluids and Structures 26, 900-917.

Gao, D., Chen, G., Chen, W., Huang, Y., Li, H., 2018. Active control with windward suction and leeward blowing. Experiments in Fluids, submitted .

Gao, D.L., Chen, W.L., Li, H., Hu, H., 2017a. Flow around a circular cylinder with slit. Experimental Thermal and Fluid Science 82, 287-301.

Gao, D.L., Chen, W.L., Li, H., Hu, H., 2017b. Flow around a slotted circular cylinder at various angles of attack. Experiments in Fluids 58, 132.

Liu, Y.G., Feng, L.H., 2015. Suppression of lift fluctuations on a circular cylinder by inducing the symmetric vortex shedding mode. Journal of Fluids and Structures 54, $743-759$.

Meyer, K.E., Pedersen, J.M., Özcan, O., 2007. A turbulent jet in crossflow analysed with proper orthogonal decomposition. Journal of Fluid Mechanics 583, 199-227.

Oruç, V., Akilli, H., Sahin, B., 2016. Piv measurements on the passive control of flow past a circular cylinder. Experimental Thermal and Fluid Science 70, 283-291. 
Pacheco, B.M., Fujino, Y., 1993. Keeping cables calm. Civil Engineering 63, 56.

Park, J., Derrandji-Aouat, A., Wu, B., Nishio, S., Jacquin, E., 2008. Uncertainty analysis: particle imaging velocimetry, in: ITTC Recommended Procedures and Guidelines, International Towing Tank Conference.

Shi, X.D., Feng, L.H., 2015. Control of flow around a circular cylinder by bleed near the separation points. Experiments in Fluids 56, 214.

Sirovich, L., 1987. Turbulence and the dynamics of coherent structures. i. coherent structures. Quarterly of applied mathematics $45,561-571$.

Zdravkovich, M., 1981. Review and classification of various aerodynamic and hydrodynamic means for suppressing vortex shedding. Journal of Wind Engineering and Industrial Aerodynamics 7, 145-189.

Zhou, B., Wang, X., Guo, W., Gho, W.M., Tan, S.K., 2015. Control of flow past a dimpled circular cylinder. Experimental Thermal and Fluid Science 69, $19-26$.

Zuo, D., Jones, N.P., 2010. Interpretation of field observations of wind- and rain-wind-induced stay cable vibrations. Journal of Wind Engineering and Industrial Aerodynamics 98, 73 - 87 . 
- The effects of active wake-jets on the suppression of aerodynamic forces and the manipulation of wake flow evolution behind a cylinder are investigated at a subcritical Reynolds number.

- Pressure measurement results demonstrate that the lift force acting on the test model is greatly reduced and drag decreased with the control of active wake-jets.

- A higher jet momentum coefficient contributes to a better control effectiveness in unsteady lift forces but not necessarily a better drag reduction.

- the active jet control scheme imposes steady and symmetric perturbations into the unsteady and asymmetric cylinder flows and the vortex shedding pattern behind the controlled cylinder is greatly modified, vortex formation length significantly elongated and the fluctuations of aerodynamic forces conceivably suppressed. 